\title{
ULTRA-LOW AMPLITUDE VARIABLES IN THE LARGE MAGELLANIC CLOUD—CLASSICAL CEPHEIDS, POP. II CEPHEIDS, RV TAU STARS, AND BINARY VARIABLES
}

\author{
J. Robert Buchler ${ }^{1,2}$, Peter R. WoOD ${ }^{2}$, AND Igor Soszyński ${ }^{3}$ \\ ${ }^{1}$ Physics Department, University of Florida, Gainesville, FL 32611, USA; buchler@phys.ufl.edu \\ 2 Research School of Astronomy \& Astrophysics, Australian National University, Canberra, Australia; wood@mso.anu.edu.au \\ ${ }^{3}$ Warsaw University Observatory, Al. Ujazdowskie 4, 00-478 Warszawa, Poland; soszynsk@ astrouw.edu.pl \\ Received 2008 September 30; accepted 2009 April 13; published 2009 May 26
}

\begin{abstract}
A search for variable stars with ultra-low amplitudes (ULAs), in the millimagnitude range, has been made in the combined MACHO and OGLE databases in the broad vicinity of the Cepheid instability strip in the HR diagram. A total of 25 singly periodic and 4 multiply periodic ULA objects have been uncovered. Our analysis does not allow us to distinguish between pulsational and ellipsoidal (binary) variabilities, nor between Large Magellanic Cloud (LMC) and foreground objects. However, the objects are strongly clustered and appear to be associated with the pulsational instability strips of LMC Pop. I and II variables. When combined with the ULA variables of Buchler et al., a total of 20 objects fall close to the classical Cepheid instability strip. However, they appear to fall on parallel period-magnitude (PM) relations that are shifted to slightly higher magnitude which would confer them a different evolutionary status. Low-amplitude RV Tauri and Pop. II Cepheids have been uncovered that do not appear in the MACHO or OGLE catalogs. Interestingly, a set of binaries seem to lie on a PM relation that is essentially parallel to that of the RV Tauri/Pop. II Cepheids.
\end{abstract}

Key words: Cepheids - instabilities - Magellanic Clouds - stars: oscillations

Online-only material: color figures

\section{INTRODUCTION}

In a previous paper (Buchler et al. 2005), we searched for periodic variability in the light curves of selected Large Magellanic Cloud (LMC) stars in MACHO Field 77. The stars were required to lie in and around the Cepheid instability strip in the HR diagram. We found seven stars with a periodic behavior of less than $0.006 \mathrm{mag}$, and labeled them ultra-low amplitude (ULA) Cepheids.

In this paper, we extend our analysis to all stars that are common to the Optical Gravitational Lensing Experiment (OGLE) II and III (Udalski et al 1999) and the MACHO (Alcock et al. 1999) databases. The stellar selection is defined in terms of the OGLE stars that are located in a parallelogram in the HR diagram defined by $14<V<17.5$ and $18.8<V+9.18(V-I)<25.0$. This region has been chosen by visual inspection to include the instability strip and colors 0.15 blueward and redward. It contains a mixture of non-oscillatory giants of spectral type F, and variable stars such as Cepheids, W Vir stars, and ellipsoidal variables and other binaries. The region has been converted from Johnson $V$ and Cousins $R_{\mathrm{c}}$ into MACHO blue magnitude $\left(M_{\mathrm{B}}\right)$ and red magnitude $\left(M_{\mathrm{R}}\right)$ using the transforms given in Alcock et al. (1999). Position-matched stars were examined in both the MACHO and OGLE databases.

The standard LMC Cepheids have already been identified both in the MACHO, EROS and the OGLE databases separately for amplitudes greater than about 0.01 mag (Beaulieu et al. 1995; Welch et al. 1995; Udalski et al 1999; Kanbur et al. 2003). Our goal here is to concentrate on ULA objects.

Fourier analysis is known to be very good at detecting periodicity in data sets even in the presence of large noise. We have therefore performed a Fourier analysis of the light curves in the combined database in the three bands MACHO $M_{\mathrm{R}}$ and $M_{\mathrm{B}}$ and OGLE I with the MUFRAN code (multifrequency analysis;
Kolláth 1990). The OGLE V band data set is generally sparse and less useful in the detection of ULA variables. The analysis is performed in the $0-0.99$ day $^{-1}$ frequency range, but the plotted frequency range in the subsequent figures is 0.004-0.99 day ${ }^{-1}$, so as to avoid both the yearly and the daily aliases. One has to be careful not to miss a potential longer period that can occur in some ellipsoidals (Mennickent et al. 2003). We have shifted obvious outlying points from the light curves prior to analysis. In this paper, all quoted amplitudes refer to Fourier amplitudes. They are determined with MUFRAN with a sinusoidal linear least-squares fit to the light curve using the frequency from the dominant Fourier peak.

We first reduce the set of over 3000 objects to those in which there are coincidences among the eight largest Fourier peaks in $M_{\mathrm{R}}$ and OGLE I. For the standard, large amplitude Cepheids, the peaks are very sharp, and these variable stars are thus readily identified. In the case of the ULA stars, the Fourier peak $M_{\mathrm{R}}$ amplitudes fall well below $0.01 \mathrm{mag}$, but nevertheless there are coincidences among the $M_{\mathrm{R}}$ and $I$ peaks. Each of these cases has to be examined individually to ascertain that the detected variability is not spurious. For this purpose, we also use the $M_{\mathrm{B}}$ data.

We mention in passing that the typical $M_{\mathrm{R}}, M_{\mathrm{B}}$, and OGLE I window functions have a very large sharp central peak with yearly side peaks at least $45 \%$ lower, but otherwise are very dull.

We note one peculiarity with the Fourier spectra which is a result of the extremely large 1 day alias of the data: all significant peaks of frequency $f$ occur with an alias at $1-f$, i.e., with peaks placed symmetrically around the frequency 0.5 day $^{-1}$. The peaks can be of comparable amplitude. The ambiguity of the period determination can generally be resolved by a comparison of the peaks in $M_{\mathrm{R}}, M_{\mathrm{B}}$, and OGLE I and V. When the symmetrically placed peak is absent, which happens when the original peak is barely above the noise, it likely is an indication that the frequency is spurious. 


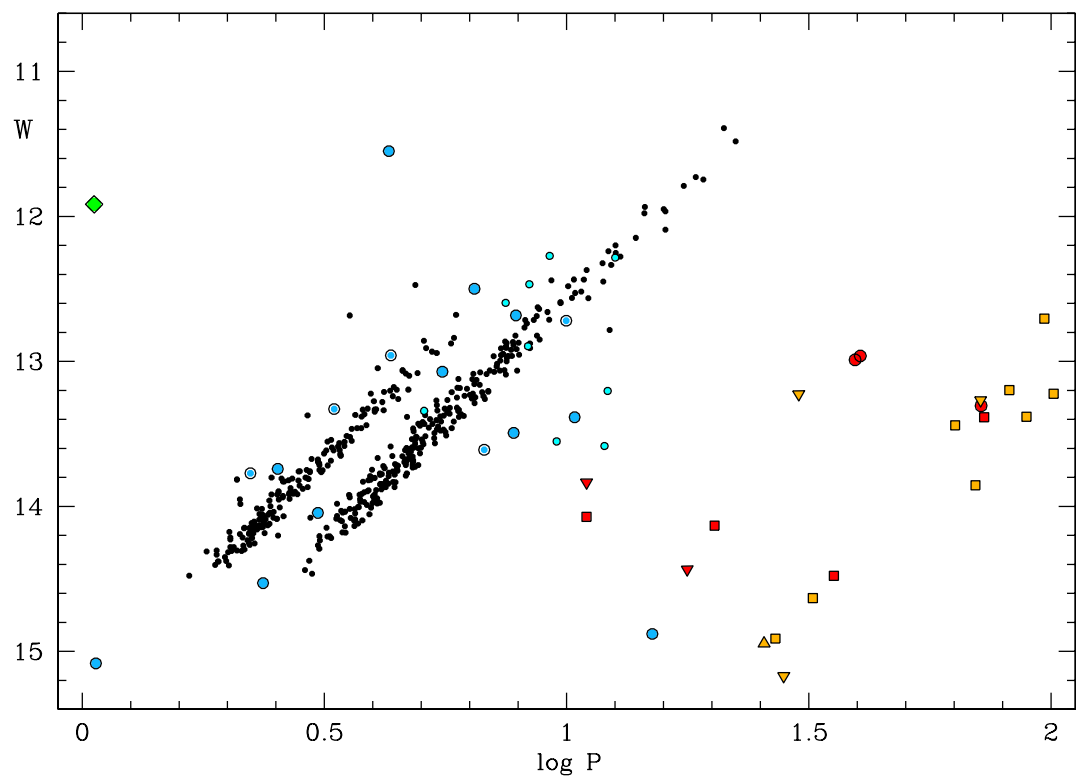

Figure 1. Period- $W$ plot for all singly periodic variables of all amplitudes: ULA variables (light blue and cyan), classical Cepheids (black dots); other larger amplitude objects categorized by light-curve shape: nonalternating (upside down triangles), regularly alternating (squares), semiregular alternations (circles), ULA alternating (triangle); Pop. II variable stars (red), binaries (orange); special object (green diamond). See the text for further details.

(A color version of this figure is available in the online journal.)

The objects we have found have been grouped into a singly periodic and a multiperiodic set, and will present them separately.

\section{SINGLY PERIODIC OBJECTS}

Figure 1 displays all the singly periodic variables that we have uncovered in a period-magnitude (PM) diagram. $W=$
$I-1.55(V-I)$ is the Wesenheit function, a reddening-free magnitude estimate. We now attempt to identify the nature of these objects.

The black dots in Figure 1 fall on two parallel bands which correspond to the fundamental and overtone classical Cepheids. They are included in the figures for reference purposes. All these Cepheids have $I$-band amplitudes greater than $\sim 0.015$ mag.

Table 1

Singly Periodic ULA Objects

\begin{tabular}{|c|c|c|c|c|c|c|c|c|c|}
\hline OGLE & MACHO & $I$ & $(V-I)$ & $P_{R}$ & $f_{R}$ & $A_{R}$ & $A_{I}$ & Band & Note \\
\hline SC6 57397 & 78.6463 .64 & 15.220 & 0.444 & 2.3629 & 0.42320 & 0.0102 & 0.0091 & RBI & \\
\hline SC8 76141 & 79.5746 .15 & 13.994 & 0.846 & 7.8600 & 0.12723 & 0.0090 & 0.0057 & RBI & \\
\hline SC11 325504 & 79.4779 .17 & 13.772 & 0.821 & 6.4537 & 0.15495 & 0.0027 & 0.0028 & RBI & \\
\hline SC15 5854 & 17.3199 .23 & 15.496 & 0.267 & 1.0674 & 0.93685 & 0.0080 & 0.0115 & RBI & \\
\hline SC13 223889 & 1.4295 .12 & 14.452 & 0.891 & 5.5415 & 0.18046 & 0.0030 & 0.0039 & RBI & \\
\hline SC1 335392 & 81.8881 .40 & 14.679 & 0.835 & 10.3895 & 0.09625 & 0.0036 & 0.0028 & RBI & \\
\hline SC1 330647 & 81.8758 .16 & 15.307 & 0.276 & 15.029 & 0.06654 & 0.0036 & 0.0030 & RBI & \\
\hline SC13 178852 & 19.4299 .298 & 14.896 & 0.745 & 2.5337 & 0.39467 & 0.0027 & 0.0038 & RI & $x$ \\
\hline SC5 416555 & 77.7189 .9 & 14.161 & 0.431 & 7.7750 & 0.12862 & 0.0026 & 0.0019 & RI & $x$ \\
\hline SC15 196983 & 1.3568 .30 & 14.855 & 0.523 & 3.0663 & 0.32613 & 0.0029 & 0.0016 & BI & $x$ \\
\hline SC2 6920 & 7.8026 .17 & 13.946 & 0.871 & 7.4876 & 0.13355 & 0.0042 & 0.0035 & RBI & $\dagger$ \\
\hline SC19 64022 & 12.10317 .10 & 13.783 & 0.975 & 9.2242 & 0.10841 & 0.0033 & 0.0043 & RBI & $\dagger$ \\
\hline SC9 127569 & 79.5378 .48 & 14.818 & 0.817 & 9.5378 & 0.10485 & 0.0035 & 0.0029 & RBI & $\dagger$ \\
\hline SC11 300879 & 79.4775 .9 & 13.654 & 0.884 & 12.600 & 0.07937 & 0.0063 & 0.0067 & RBI & $\dagger$ \\
\hline SC14 7197 & 1.3561 .6 & 14.580 & 0.799 & 5.0807 & 0.19682 & 0.0048 & 0.0045 & RBI & $\dagger$ \\
\hline SC7 344553 & 80.6351 .14 & 13.886 & 0.915 & 8.3764 & 0.11938 & 0.0049 & 0.0025 & RBI & $\dagger$ \\
\hline SC20 181340 & 12.10922 .11 & 14.873 & 0.832 & 11.970 & 0.08354 & 0.0049 & 0.0040 & RBI & $\dagger$ \\
\hline SC21 140323 & 6.6697 .11 & 14.136 & 0.801 & 8.3250 & 0.12012 & 0.0041 & 0.0030 & RBI & $\dagger$ \\
\hline SC13 191166 & 19.4302 .323 & 14.450 & 0.804 & 12.1561 & 0.08226 & 0.0032 & 0.0029 & RBI & $x \dagger$ \\
\hline SC2 357794 & 81.8392 .8 & 13.109 & 1.006 & 4.2964 & 0.23275 & 0.0059 & 0.0013 & S RBI & \\
\hline SC4 296029 & 77.7428 .36 & 14.819 & 0.676 & 2.2250 & 0.44953 & 0.0024 & 0.0022 & RBI & $*$ \\
\hline SC4 323401 & 77.7430 .18 & 14.505 & 0.759 & 3.3116 & 0.30187 & 0.0042 & 0.0033 & RI & $*$ \\
\hline SC3 153959 & 77.7789 .25 & 14.116 & 0.901 & 9.9817 & 0.10018 & 0.0045 & 0.0034 & RBI & $*$ \\
\hline SC3 35239 & 77.7668 .981 & 14.149 & 0.768 & 4.3373 & 0.23056 & 0.0054 & 0.0032 & RI & $*$ \\
\hline SC4 176301 & 77.7306 .43 & 14.566 & 0.617 & 6.7609 & 0.14791 & 0.0109 & 0.0025 & RBI & $*$ \\
\hline
\end{tabular}

Notes. Columns: OGLE and MACHO IDs, $I$-band magnitude, color, period, frequency, $M_{\mathrm{R}}$ and OGLE I band Fourier amplitudes. See the text for Columns 9 and 10. Object SC2 357794 lies considerably above the Cepheid PM relation $(W, \log P)$ and is a strange Cepheid candidate. 


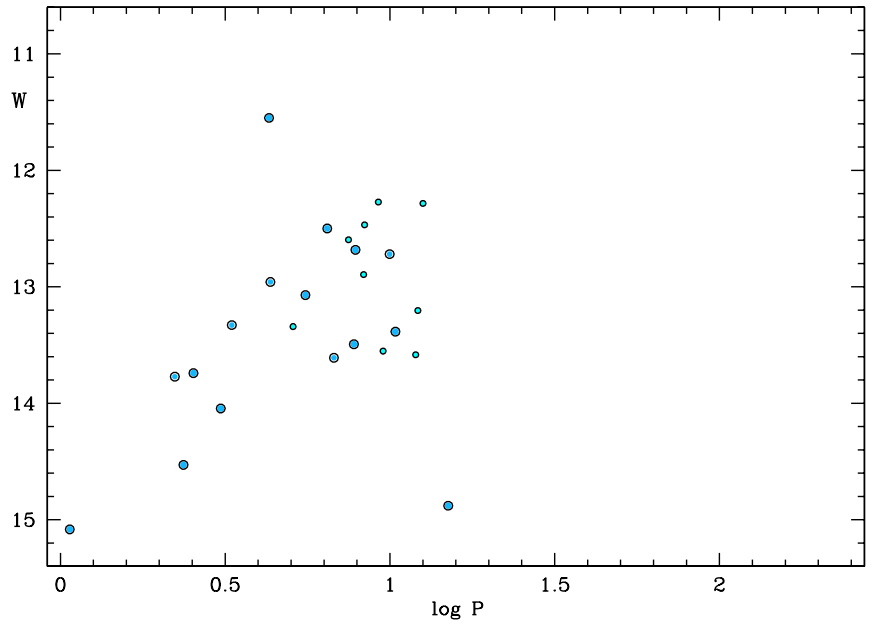

Figure 2. Top: period- $W$ plot of all singly periodic ULA variables in the searched period range, showing a clear clustering in the Cepheid instability strip.

(A color version of this figure is available in the online journal.)

The red and orange symbols below the classical Cepheids have relatively large amplitudes. We will return to them below.

The 25 ULA variables we have found are listed in Table 1. All objects have I-band amplitudes less than 0.0067, except for SC15 5854 which has an amplitude of 0.0115 . The first two columns of Table 1 give the MACHO and OGLE names, respectively, followed by the average $I$ magnitude and $(V-I)$ color, and the period, $P_{R}$, frequency $F_{R}$, and the Fourier amplitudes in $M_{\mathrm{R}}$ and in OGLE I. The frequency $f_{R}$ (and period) is optimized in a linear squares Fourier fit to the $M_{\mathrm{R}}$ data starting with the highest $M_{\mathrm{R}}$ spectral peak.

The penultimate column denotes the bands in which there is a strong common peak in the Fourier spectra. By strong we mean that its amplitude stands out by at least a factor of 2 above the noise level. The asterisk in the last column denotes stars that were already discussed by Buchler et al. (2005).

We have plotted the ULA variables as light blue and cyan circles. For the four ULA objects that are branded with an $\times$, the evidence for very low amplitude periodicity is a little less strong in the sense that it does not satisfy all our criteria. We will return to them shortly.

Because of the observational noise, we have no way of distinguishing ULA pulsating stars from ellipsoidal variables. However, we shall argue that there are very few ellipsoidals in our sample. For that purpose, we present Figure 2 which locates, unencumbered, only the ULA variables in the whole range we have analyzed, namely the $W$ range corresponding to the chosen parallelogram in the HR diagram (see Section 1) and the frequency range from 0.004 to 0.99 days $^{-1}$, i.e., $\log P$ [days] $=0.0044-2.40$. The way the ULA objects cluster near the instability strip in the PM diagram suggests that a large majority of the objects are intrinsic variables and are associated with either the classical Cepheids or, the lower lying ones, the Pop. II Cepheids.

In the OGLE data (Udalski et al 2008), each frame was processed with the DoPHOT photometry program. In this way, independent point-spread function (PSF) profile photometry of each object was derived, supplemented with astrometric information (current $X, Y$ position) obtained via PSF fitting. As a result, proper motions can be inferred. In fact, unpublished OGLE-III data suggest that nine objects show proper motion, which in turn suggests that they are foreground stars. They are represented as smaller cyan filled circles in Figures 1 and 2, and are branded with a dagger in column 10 of Table 1 . However, if they are indeed foreground stars in the solar vicinity that overlap in the color-magnitude diagram (CMD) with the LMC instability strip (Soszyński et al. 2002), it is nevertheless curious that six of them, additionally, appear so near the LMC classical Cepheid PM relations, and the other three clusters just below.

If they are foreground stars, one would think that they are some sort of main-sequence ellipsoidal binary in the solar vicinity. Generally, spectra can be used to tell giants from dwarfs and to derive radial velocities, so potentially foreground or LMC status could be investigated by spectral observations, along with radial velocity variations to check binarity versus pulsation.

For completeness, we note that we have also found one egregious large amplitude variable in the database, viz., SC8 81586 (79.5626.10). Despite the relative sparsity of its OGLE and MACHO data and the noisiness of its MACHO data, this object has a sharply defined period of $P=1.0589$ days, and relatively large amplitudes $\left(A_{R}=0.024, A_{I}=0.025\right.$, $\left.A_{B}=0.036\right)$. It is depicted as a green diamond in the upper left side in Figure 1.

\subsection{Fourier Spectra and Light Curves of the Singly Periodic Ultra-low Amplitude (ULA) Objects}

Plots of the results of our Fourier analysis of each of these objects would take up too much space in this paper. We therefore give just a few representative examples.

Our figures are in a standard format and contain (1) panels with the amplitude Fourier spectra for the $M_{\mathrm{R}}, M_{\mathrm{B}}$, and OGLE I, respectively, plotted for a frequency range of 0.0040.99 days $^{-1}$. (2) Panels with the actual data points on the bottom right; because the OGLE observations start three and 3/4 years (1376.5 days) after the MACHO observations, we have shifted them backward in time in the OGLE panel for better visualization. (3) Panels on the top right side that display the light curves folded with the period that is indicated on top of the figure; the MACHO and OGLE data have been phased by taking the 1376.5 day shift into account. The solid red curves in the top right panels represent single frequency harmonic fits to the phased and folded $M_{\mathrm{R}}, M_{\mathrm{B}}$, and OGLE I data. In some cases, we have made small adjustments to the folding period $f_{R}$ to improve the phasing.

The analyses of SC8 76141, SC14 7197, and SC11 325504 are exhibited in Figures 3-5. They are among the top 16 objects of Table 1 and they satisfy all our criteria for variability, viz., clear peaks in all three bands, a strong 1 day alias, good consistent phasing of the folded light curves.

Finally, we wish to give two examples of variability at the confidence limit. Figure 6 displays the analysis of SC13 178852. The OGLE I data have a very convincing spectrum with a dominant peak at 0.3947 days $^{-1}$. While the corresponding peak is indeed the largest in $M_{\mathrm{R}}$ and $M_{\mathrm{B}}$, one would perhaps not have claimed variability on the basis of the MACHO or the OGLE data alone. However, it is unlikely that the same object would show common peaks, however weak if the variability were not real. Note also that the $M_{\mathrm{R}}$ spectrum shows the $1-f$ alias and that there is a strong hint of it in the OGLE I spectrum. Furthermore, the folded light curves show in-phase variability.

In Figure 7, we present perhaps our weakest case (SC15 196983) for variability. OGLE I and $M_{\mathrm{B}}$ have their dominant peak at $f_{\mathrm{o}}=0.3261$ days $^{-1}$. OGLE I has a strong 1 day alias 


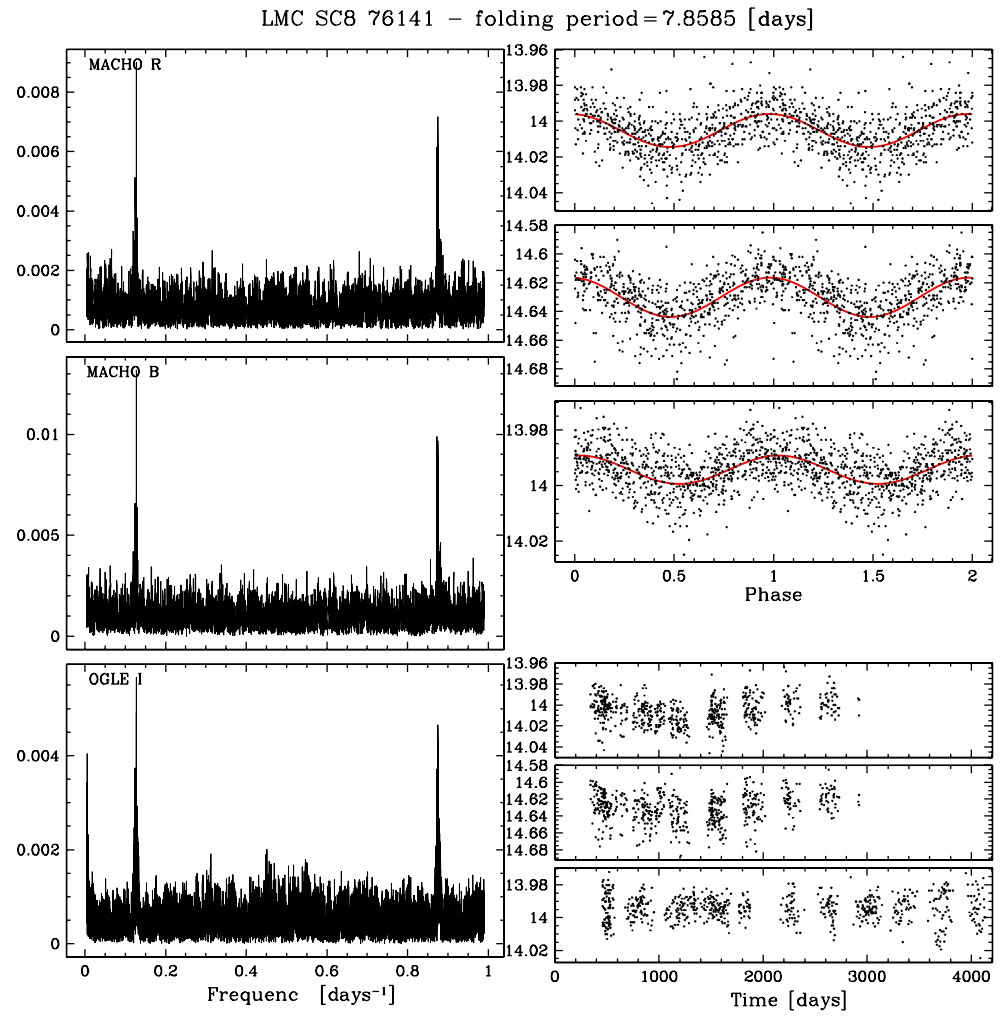

Figure 3. LMC SC8 76141—left side, top down: Fourier amplitude spectra of the $M_{\mathrm{R}}, M_{\mathrm{B}}$, and OGLE I data; right side, top: folded and phased data; folding period appears on top; bottom: corresponding data points.

(A color version of this figure is available in the online journal.)
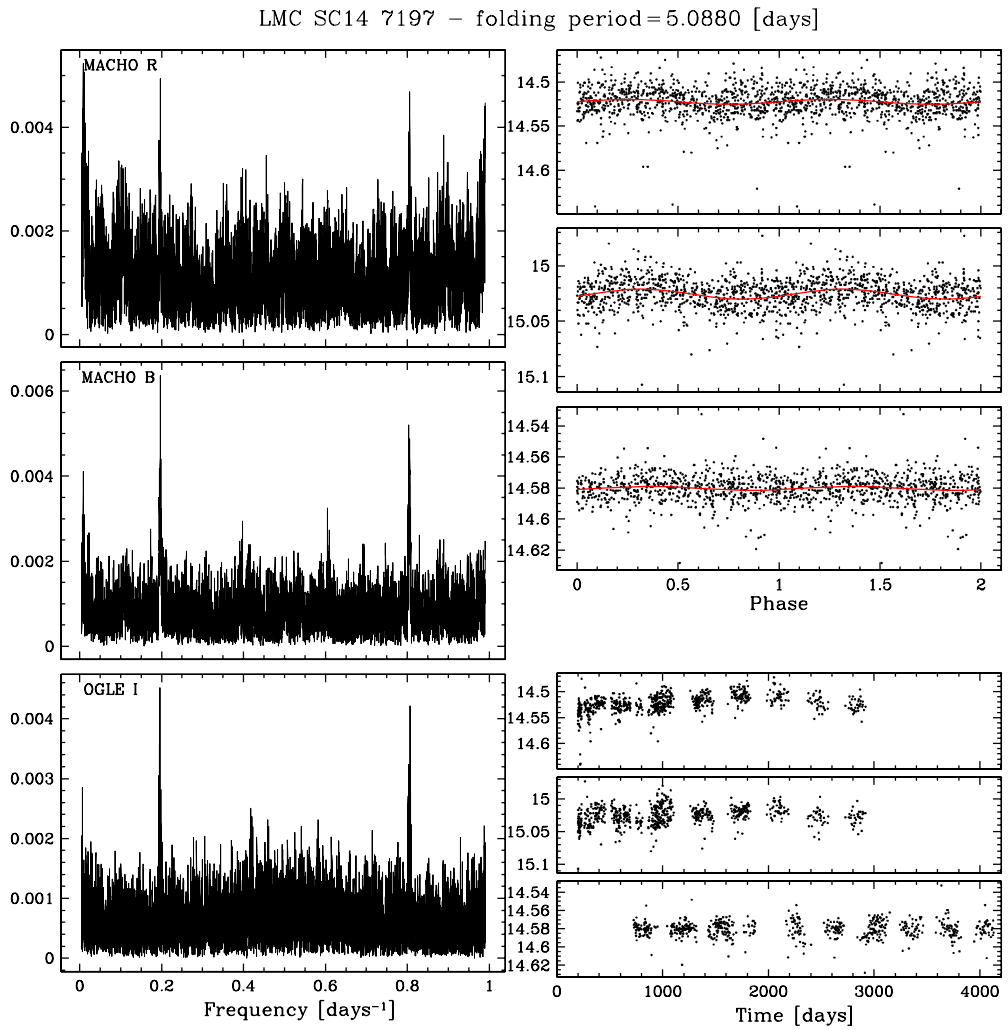

Figure 4. LMC SC14 7197—left side, top down: Fourier amplitude spectra of the $M_{\mathrm{R}}, M_{\mathrm{B}}$ and OGLE I data; right side, top: folded and phased data; folding period appears on top; bottom: corresponding data points.

(A color version of this figure is available in the online journal.) 

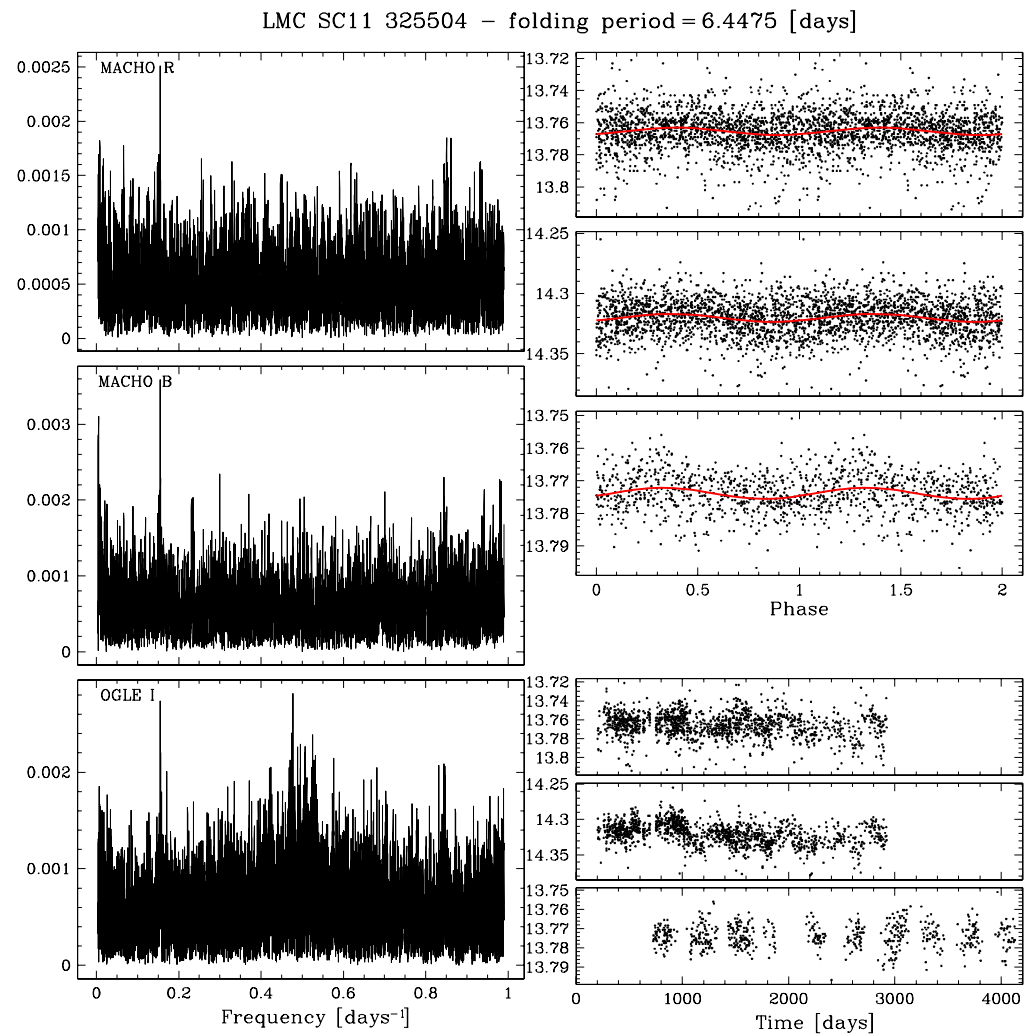

Figure 5. LMC SC11 325504—left side, top down: Fourier amplitude spectra of the $M_{\mathrm{R}}, M_{\mathrm{B}}$, and OGLE I data; right side, top: folded and phased data; folding period appears on top; bottom: corresponding data points.

(A color version of this figure is available in the online journal.)
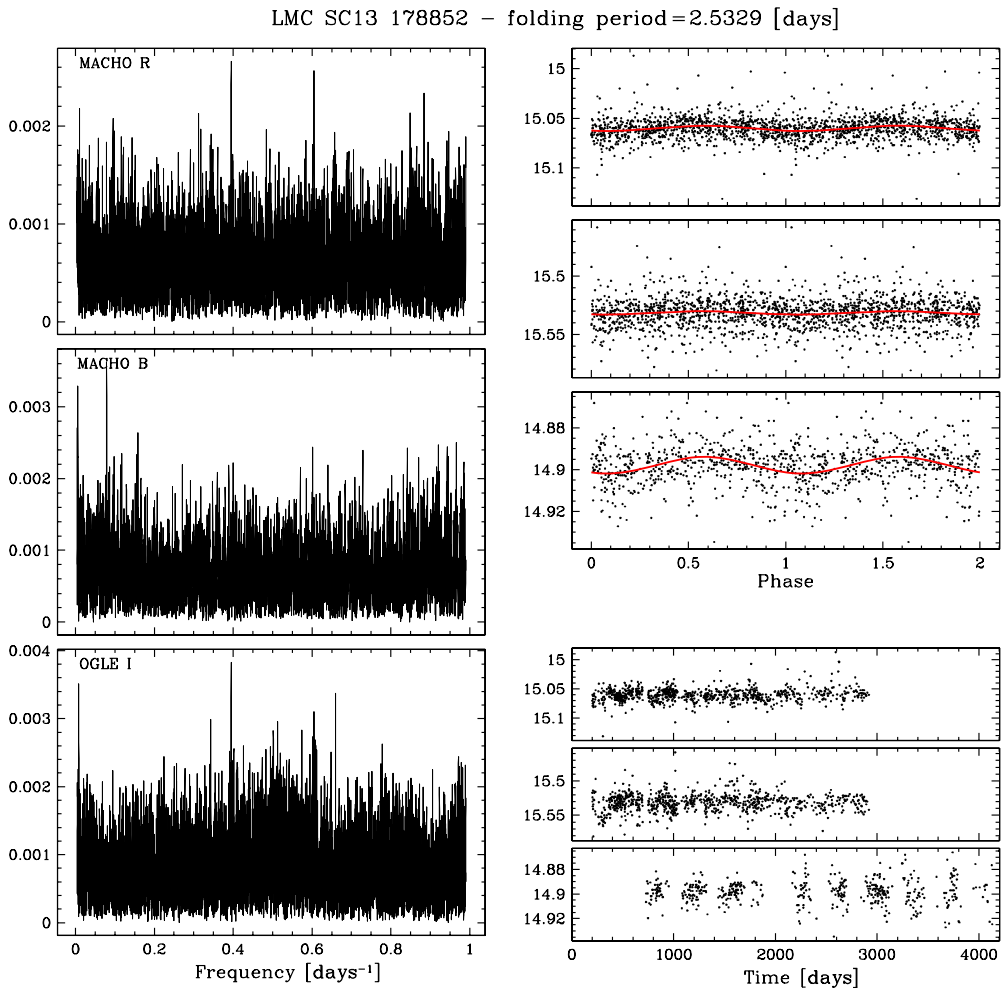

Figure 6. LMC SC13 178852 - left side, top down: Fourier amplitude spectra of the $M_{\mathrm{R}}, M_{\mathrm{B}}$, and OGLE I data; right side, top: folded and phased data; folding period appears on top; bottom: corresponding data points.

(A color version of this figure is available in the online journal.) 
LMC SC15 196983 - folding period = 3.0663 [days]
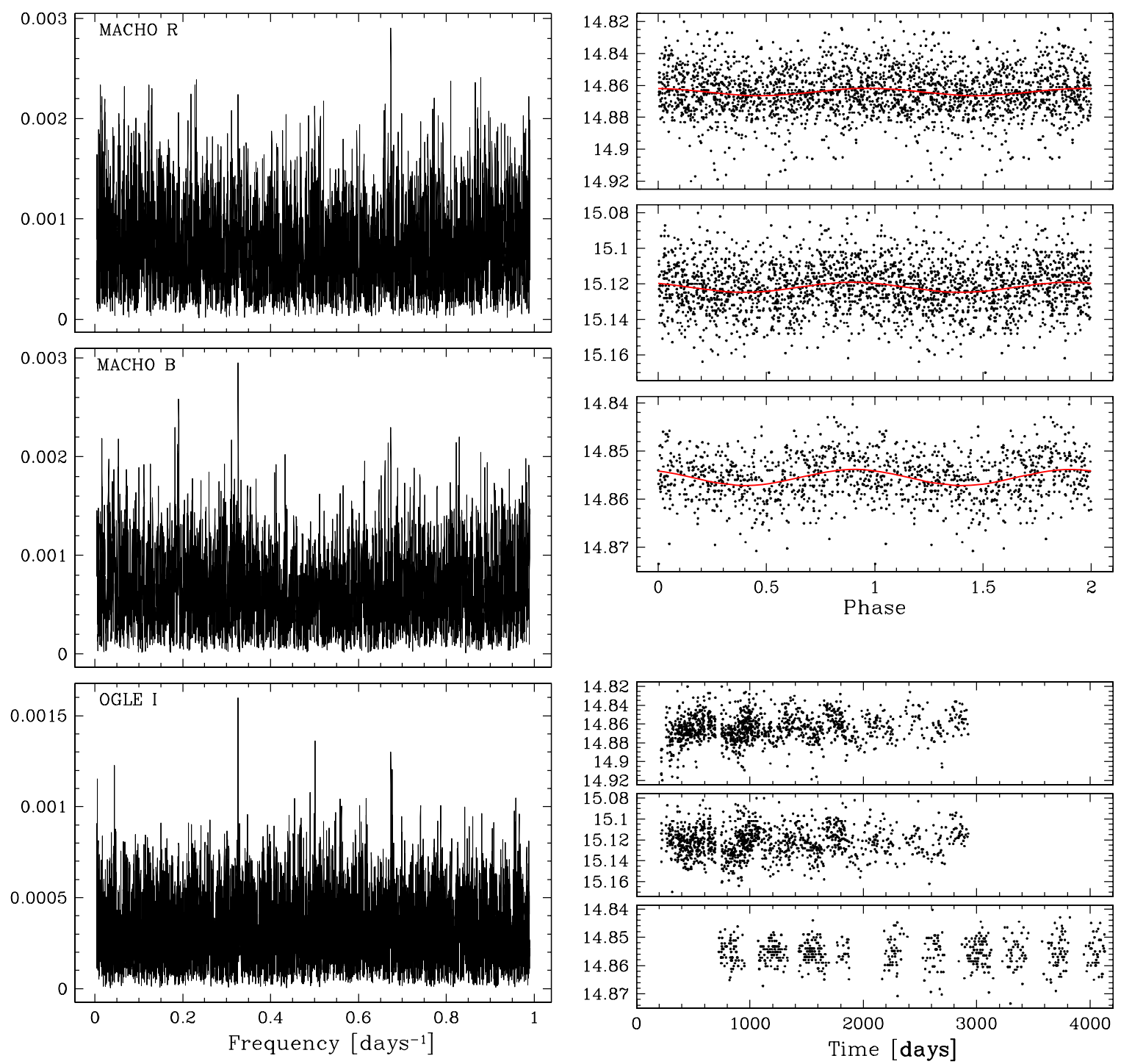

Figure 7. LMC SC15 196983-left side, top down: Fourier amplitude spectra of the $M_{\mathrm{R}}, M_{\mathrm{B}}$, and OGLE I data; right side, top: folded and phased data; folding period appears on top; bottom: corresponding data points.

(A color version of this figure is available in the online journal.)

peak at 0.6739 , which is also present in $M_{\mathrm{B}}$ albeit just barely. However, this is the prominent peak in $M_{\mathrm{R}}$ where the $f_{\mathrm{o}}$ peak is hidden in the "grass." We have chosen the $f_{\mathrm{o}}$ peak as the actual frequency of this object for two reasons: first because it is the dominant peak in the OGLE I data which have the cleanest spectrum, and second because the other peak gives a bad phasing between the folded MACHO and OGLE data.

\subsection{Discussion}

Since many of the objects lie in crowded fields, the question arises as to the reliability of our assignments. The variability analysis and the periods that have been determined are immune to contamination by neighboring objects unless those objects have variability themselves or our object is part of a binary, for example.

On the other hand, contamination by a neighboring object will affect the magnitude and the color of our objects. We have looked in some detail at the three objects SC4 62503, SC5 124597, and SC4 295930, for example. The first two are isolated objects, but the third one is a messy clump, so it is possible (but not necessary) that the photometry of this last object is poor. This will apply to both the OGLE and MACHO data. $V-I$ for this object is 1.38 , way outside the typical range (see the CM plot of Figure 8). A further look at SC4 295930 in $I-M_{\mathrm{R}}, V-M_{\mathrm{B}}$, and $V-I$ reveals that $I$ is anomalously bright, by at least $0.5 \mathrm{mag}$. Consequently, it should be shifted down in the PM plot and could well be a regular classical Cepheid of very low amplitude rather than a strange Cepheid.

We now turn to Figure 8. The top panel is a blowup of the PM $(\log P, W)$ plot of Figure 1, and the bottom panel is the corresponding $\mathrm{CM}(V-I, I)$ plot. All the objects that are listed in Table 1 and in Buchler et al. (2005) are identifiable in the figure. The squares, diamonds, filled circles, and upright triangles indicate the newly found ULAs. The filled circles locate the potential foreground objects. The $\times \mathrm{s}$ denote the five variables that are in common with the previous work of Buchler et al. (2005) which concentrated on MACHO Field 77. The crosses are their additional variables that lie marginally outside 

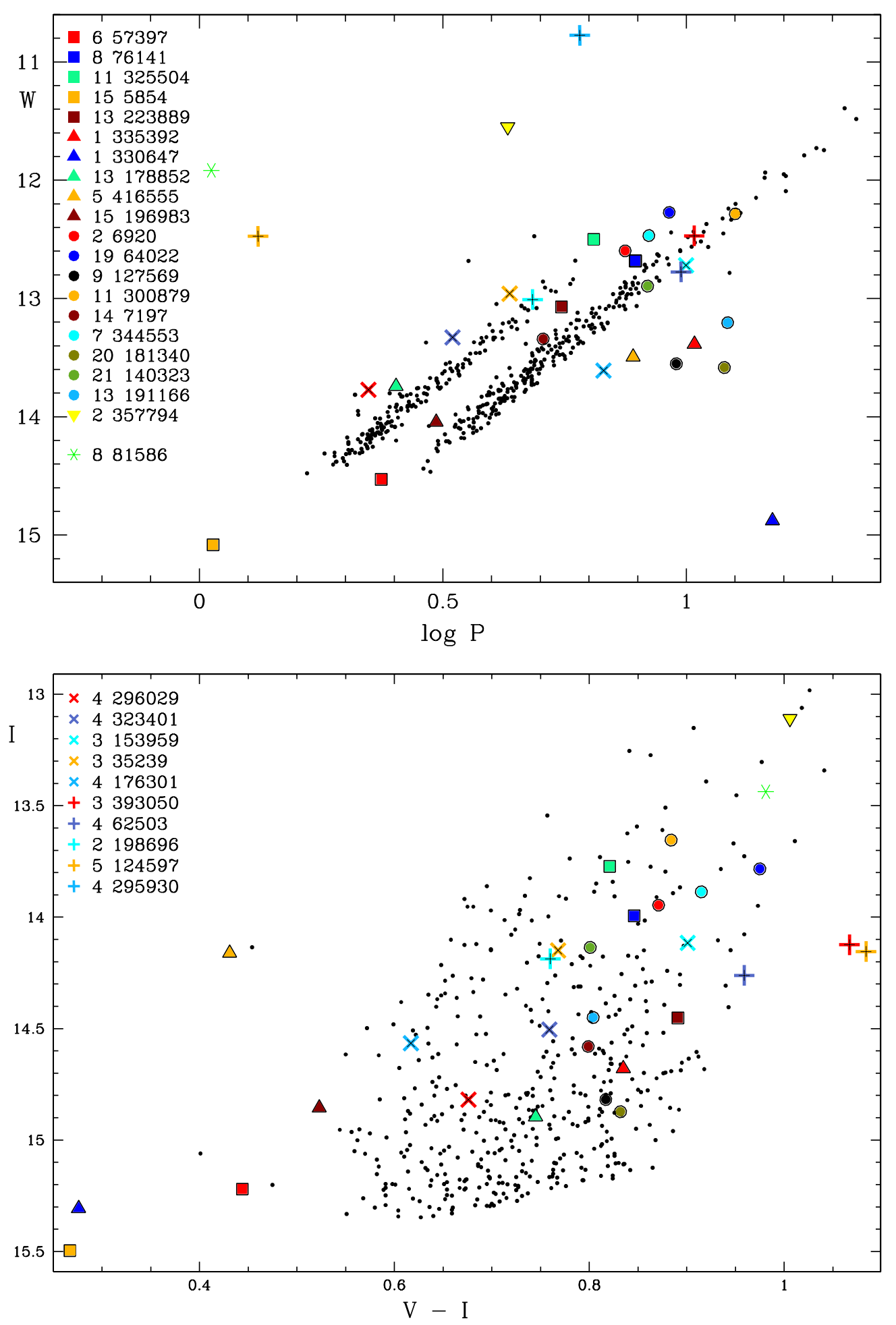

Figure 8. Top: period- $W$ plot for ULA variables (large symbols), where $W=I-1.55(V-I)$. Also shown are the classical Cepheids (small black dots) from our combined MACHO-OGLE database. Bottom: $I, V-I$ plot for the same stars. The black dots represent the classical Cepheids. The five squares, five triangles, and nine filled circles mark the position of the ULA Cepheids, the upside-down triangle that of the potential strange Cepheid candidate SC2 357794. The crosses and $\times \mathrm{s}$ denote the objects from Buchler et al. (2005). SC4 295930 has a $V-I=1.38$, outside the plotted range. The green asterisk in the CDM locates the egregious variable star SC8 81585 that is located in the upper left corner in Figure 1.

(A color version of this figure is available in the online journal.)

the cut that defines this paper's database (see Section 1). For reference, we have again plotted the classical Cepheids as small black dots.

These ULA objects are certainly a mixture of pulsating stars with perhaps a few ellipsoidals that can also have amplitudes in the millimagnitude range. Of course, the ULA Cepheids have to satisfy the known PM relations, but it is difficult to distinguish between these two types of low-amplitude variability.

One notes that 20 objects of Table 1 fall into the classical Cepheid range, in both the PM and the CM diagrams. Most of 
Table 2

Larger Amplitude Singly Periodic Objects

\begin{tabular}{|c|c|c|c|c|c|c|c|}
\hline OGLE ID & MACHO ID & $M_{I}$ & $W$ & $P$ (days) & $A_{I}$ & Note & Type of Star \\
\hline SC21 85305 & 6.6575 .25 & 15.065 & 14.071 & 10.99 & & Alt & Pop. II \\
\hline SC8 52612 & 79.5622 .20 & 14.638 & 13.835 & 10.99 & 0.038 & No alt & Pop. II \\
\hline SC7 295173 & 78.6223 .44 & 15.036 & 14.435 & 17.74 & 0.037 & No alt & Pop. II \\
\hline SC4 53483 & 77.7306 .73 & 14.902 & 14.133 & 20.21 & & Crested & Pop. II \\
\hline SC4 287444 & 77.7426 .59 & 15.288 & 14.911 & 26.97 & & Alt & Binary \\
\hline SC1 325044 & 81.8758 .27 & 15.533 & 15.170 & 28.06 & 0.015 & No alt & Binary \\
\hline SC6 462158 & 80.6710 .17 & 14.113 & 13.226 & 30.14 & 0.014 & No alt & Binary \\
\hline SC10 137113 & 2.4902 .4632 & 15.175 & 14.633 & 32.22 & & Alt & \\
\hline SC10 105184 & 79.4896 .51 & 15.225 & 14.478 & 35.61 & & Alt & Pop. II \\
\hline SC5 92493 & 78.6949 .2259 & 14.090 & 12.989 & 39.4 & & Semireg & Pop. II \\
\hline SC3 274381 & 77.7911 .4 & 14.105 & 12.963 & 40.4 & & Semireg & Pop. II \\
\hline SC14 57979 & 19.3694 .19 & 14.662 & 13.441 & 63.40 & & Alt & Binary \\
\hline SC11 338244 & 79.4659 .3417 & 14.972 & 13.854 & 69.82 & & Alt & Binary \\
\hline SC11 338203 & 79.4780 .27 & 14.569 & 13.306 & 71.7 & & Semireg & Pop. II \\
\hline SC9 263456 & 79.5504 .13 & 14.684 & 13.267 & 71.78 & 0.057 & No alt & Binary \\
\hline SC6 40874 & 78.6461 .2152 & 14.361 & 13.385 & 72.76 & & Alt & Pop. II \\
\hline SC8 181816 & 78.5856 .2363 & 14.383 & 13.197 & 81.99 & & Alt & Binary \\
\hline SC14 100796 & 1.3810 .19 & 14.038 & 13.382 & 88.95 & & Alt & Binary \\
\hline SC9 237168 & 79.5501 .13 & 13.845 & 12.706 & 96.90 & & Alt & Binary \\
\hline SC14 100798 & 1.3810 .30 & 14.323 & 13.226 & 101.26 & & Alt & Binary \\
\hline SC3 201554 & 77.7794 .46 & 15.527 & 14.9457 & 25.57 & 0.007 & Binary & \\
\hline
\end{tabular}

Notes. Columns: OGLE and MACHO IDs, I-band magnitudes, period, $I$-band Fourier amplitude, star type. In the case of alternating cycles, the period has been chosen to visually give the best folded light curve, rather than to correspond to the Fourier peak with the highest amplitude.

the ULA objects fall outside of or near the edges of the F and O1 instability strips. That ULA Cepheids would be at the edges of the instability strip is consistent with theory (Buchler \& Kollath 2002; Buchler et al. 2005).

However, one might claim that the figure indicates that the ULA objects form two separate, parallel fundamental and overtone PM relations in which case they would be physically distinct from the regular Cepheids (perhaps on the first crossing of the instability strip rather than on the second or third crossings on the blue loop). The CMD, unfortunately, has too much scatter to provide any discriminating information.

One object, SC15 5854, with a period of 1.0674 days lies in the continuation of the Cepheid PM relation and falls into the CM range of the Cepheids, albeit quite beyond the tip.

There is a cluster of six stars that falls below the Cepheid PM relation with periods 2-3 times longer than that of classical Cepheids of the same magnitude. Three of them are possibly foreground stars, as discussed in Section 2. Again, we cannot be sure that these objects are not ellipsoidals, but it seems unlikely because of the clustering in PM. Furthermore, four of them (SC9 127569, SC20 181340, SC1 335382, and SC13 191166) also cluster in CM. This suggests that they are pulsators rather than ellipsoidals. What is their nature? They are unlikely to be classical Cepheids because they have too small a brightness for their period, and potential contamination by neighboring objects would move them even lower in the PM diagram. It is possible that these stars are also ULA Population II stars. However, SC5 416555, which is very blue is perhaps an ellipsoidal.

The star SC2 357794, marked with an inverted triangle in Figure 8, falls into the proper $\mathrm{CM}$ range, but it has a period a factor of $\sim 5$ smaller than the fundamental Cepheid period, i.e., the period the star would have if it pulsated in the $F$ mode. The theoretical work of Buchler et al. (1997) has shown that some Cepheids can pulsate in a surface mode with a period that is about a factor of 4-5 shorter than the corresponding fundamental period. This object therefore is possibly a strange Cepheid (Buchler \& Kollath 2001). But one cannot rule out that the light curve might be contaminated by a bright nearby star in which case it could be a regular ULA Cepheid as we already suggested for object SC5 124597 that Buchler et al. (2005) had identified as a strange Cepheid candidate.

Object SC1 330647 is a little offset with $W \sim 15$ and log $P \sim 1.2$ and lies in the W Vir instability region (see Figure 1). It could be a ULA W Vir star although its $V-I$ color is very blue.

We conclude that most if not all of the ULA singly periodic objects in the data set are Pop. I and Pop. II Cepheids, with the caveat that some might be foreground stars or ellipsoidals.

\subsection{Other, Larger Amplitude Variable Objects}

We now turn to the larger amplitude singly periodic stars that are exhibited in Table 2 . The objects that we identify as probable binaries are marked in orange and the probable Pop. II or RV Tau in red in Figure 1.

Their identifiers, their average $I$ magnitudes, Wesenheit magnitudes $W$, and their periods (or cycling times) are given in Table 2. The first two columns give the MACHO and OGLE names. All these stars have $I$-band Fourier amplitudes in the range 0.01-0.25. An exception is the ULA SC3 201554 with harmonic peak amplitudes of 0.0065 and 0.0075 that is shown in yellow. The objects that are displayed as triangles have regular light curves. One object, SC4 53483, has a "crested top" light curve that is typical of some $\mathrm{W}$ Vir stars. The remainder show behavior with cycle-to-cycle alternations. These alternations are regular (squares) for some and some semiregular (circles).

The period association is sometimes ambiguous by a factor of 2 when alternations are present. We have not chosen the period with the highest Fourier peak, but somewhat arbitrarily the one that visually gives the folded light curve with the least scatter. 
One notes that all but two points would fall on two parallel, relatively tight PM relations that are separated by 0.3 (a factor of 2 in $P$ ). The binaries (orange) fall predominantly on the right sequence, and the Pop. II/RV tau stars (red) predominantly on the left one.

Actually, from a physical point of view, for the pulsating stars the shorter period is more relevant as it corresponds to the linearly excited pulsational mode, whereas the longer period arises through period doubling that is caused by nonlinear effects (Buchler \& Kovacs 1986; Kovacs \& Buchler 1987).

For the binaries, the reverse situation holds. Here, there are also two sequences separated by 0.3 in $\log P$ because the "no alt" stars can adopt a period equal to half the orbital period (there are two maxima per orbit).

But, why does the binary sequence exist? We will argue that it is because stars on the blue loops have a certain size at a given luminosity and color, and if they have a companion that is capable of causing ellipsoidal variations, it must be at a small multiple of the stellar radius. This radius increases with luminosity $(I)$ and color $(V-I)$. The conversion to $W$ will tend to take out the $V-I$ variation along tracks at given luminosity. If we remember that for both orbits and pulsation, $P \sim R^{1.5}$, and that the period of the fundamental pulsation mode is not too different from the orbital period at the stellar surface, we can see that qualitatively the binary sequence should be parallel to the pulsation sequence: more detailed computations would be needed to estimate the exact shift in $\log P$. The stars on the binary sequence should therefore be in the LMC.

We have found low-amplitude RV Tauri and Pop. II Cepheids in the LMC that do not appear in the catalogs of (Alcock et al. 1999; Udalski et al 1999). We also find that, curiously, a set of binaries appear to lie on essentially the same PM relation as the RV Tauri/Pop. II Cepheids.

\section{MULTIPERIODIC VARIABLES}

Four of the ULA stars have multiple periods. They are displayed in the PM plot of Figure 9. Again for reference, we have superposed the Cepheids as small black dots. The bottom panel displays the corresponding $(I, V-I)$ plot. The properties of these objects appear in Table 3.

The results of the individual Fourier analyses are presented in Figures 10-13.

1. LMC SC6 248178. The results of the analysis are displayed in Figure 10. Table 3 indicates that this star has its largest frequency peak at $f_{1}=0.05819$ days $^{-1}$, i.e., 17.185 days, and two close frequencies, $f_{2}=0.36484$ and $f_{3}=$ 0.30657 days $^{-1}$. One notes that $f_{3} \sim f_{2}+f_{1}$. Even though the three amplitudes are all in the low-millimagnitude range, the three peaks stand out very clearly in $M_{\mathrm{R}}, M_{\mathrm{B}}$, and OGLE I. Peaks 2 and 3 suggest that if this star is a Cepheid it may be a higher order double-mode pulsator with a large
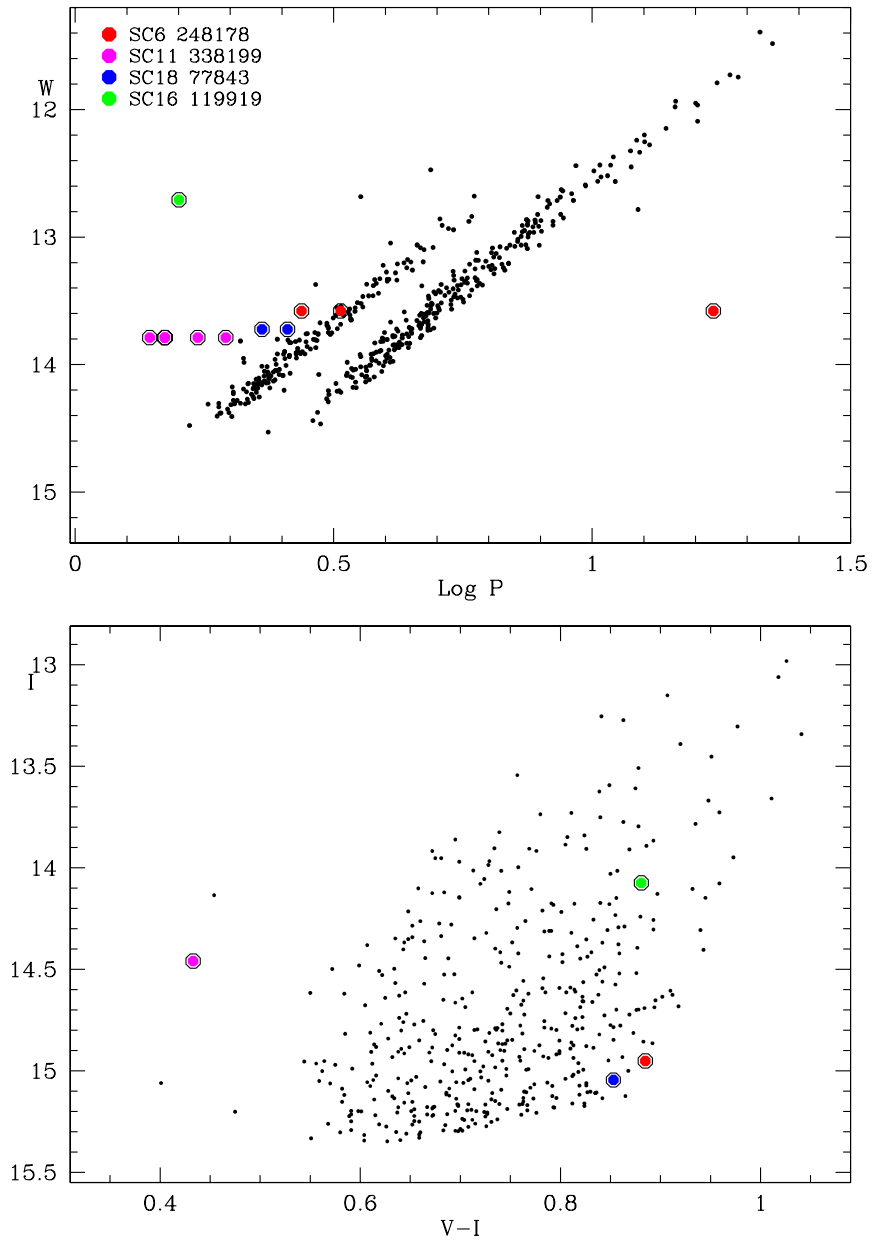

Figure 9. Top: period- $W$ magnitude plot. The objects of this paper are superposed on the Cepheids from our combined MACHO-OGLE database. The various periods of each star appear on a horizontal alignment; bottom: $(I, V-I) \mathrm{CM}$ plot.

(A color version of this figure is available in the online journal.)

period ratio of $P_{3} / P_{2}=0.84028$. If the long period of 17.185 days were due to an unseen binary companion that would not explain the observed frequency lock.

2. LMC SC18 77843. The analysis of this object appears in Figure 11. There are two peaks with a period ratio of 0.89177 which is larger than that of a double-mode Cepheid that pulsates in the $\mathrm{O} 1$ and $\mathrm{O} 2$ modes. The star also lies somewhat to the left of the PM relation, both when plotted with $I$ and with the Wesenheit magnitude $W$, consistent with the expected positions of higher order modes.

3. LMC SC16 119919. This star is a 1.59 day period variable with an extremely long period (1380 days) modulation (Figure 12). Looking at the light-curve points, one would think that we have mismatched the OGLE object with the

Table 3

ULA Multimode Stars

\begin{tabular}{lcccccccccccc}
\hline \hline OGLE & MACHO & $I$ & $(V-I)$ & $f_{1}$ & $f_{2}$ & $f_{3}$ & $f_{4}$ & $A_{1}$ & $A_{2}$ & $A_{3}$ & $A_{4}$ \\
\hline SC6 248178 & 78.6699 .27 & 14.951 & 0.885 & 0.05819 & 0.36484 & 0.30657 & & 0.0048 & 0.0034 & 0.0034 & & \\
SC18 77843 & 11.9838 .21 & 15.045 & 0.853 & 0.43528 & 0.38817 & & & 0.0050 & 0.0039 & & \\
SC16 119919 & 81.8998 .12 & 14.074 & 0.881 & 0.63018 & 0.00072 & & & 0.0048 & 0.0159 & & \\
SC11 338199 & 79.4659 .3384 & 14.459 & 0.433 & 0.67103 & 0.51146 & 0.71783 & 0.57911 & 0.0083 & 0.0070 & 0.0055 & 0.0051 & $\dagger$
\end{tabular}

Notes. Columns: OGLE and MACHO identifiers, $I$ magnitude, $V-I$ color, significant frequencies, corresponding $M_{\mathrm{R}}$ Fourier amplitudes. (The last object's amplitude is a little larger than our ULA criterion.) A dagger in the last column denotes potential proper motion. 

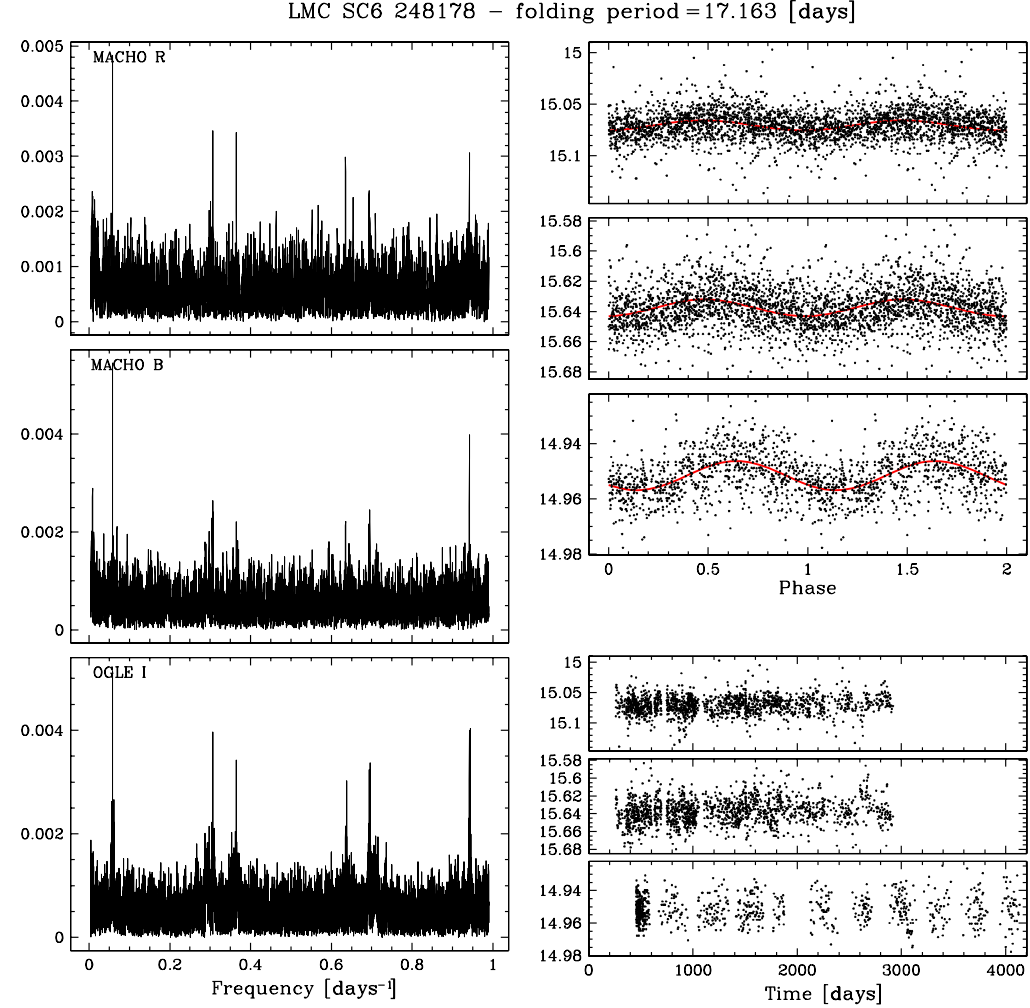

Figure 10. LMC SC6 248178—-eft side, top down: Fourier amplitude spectra of the $M_{\mathrm{R}}, M_{\mathrm{B}}$, and OGLE I data; right side, top: folded and phased data; folding period appears on top; bottom: corresponding data points.

(A color version of this figure is available in the online journal.)
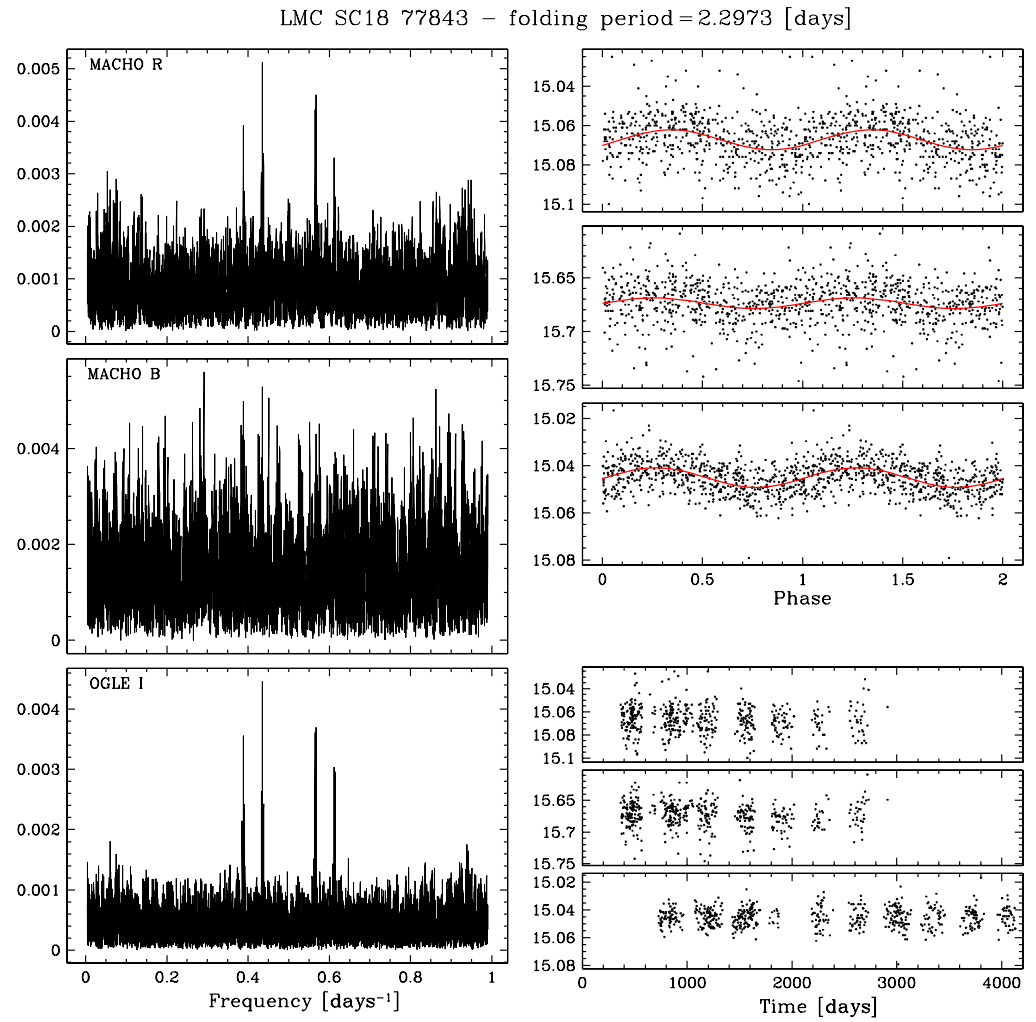

Figure 11. LMC SC18 77843-left side, top down: Fourier amplitude spectra of the $M_{\mathrm{R}}, M_{\mathrm{B}}$, and OGLE I data; right side, top: folded and phased data; folding period appears on top; bottom: corresponding data points.

(A color version of this figure is available in the online journal.) 
LMC SC16 119919 - folding period $=1.5876$ [days]
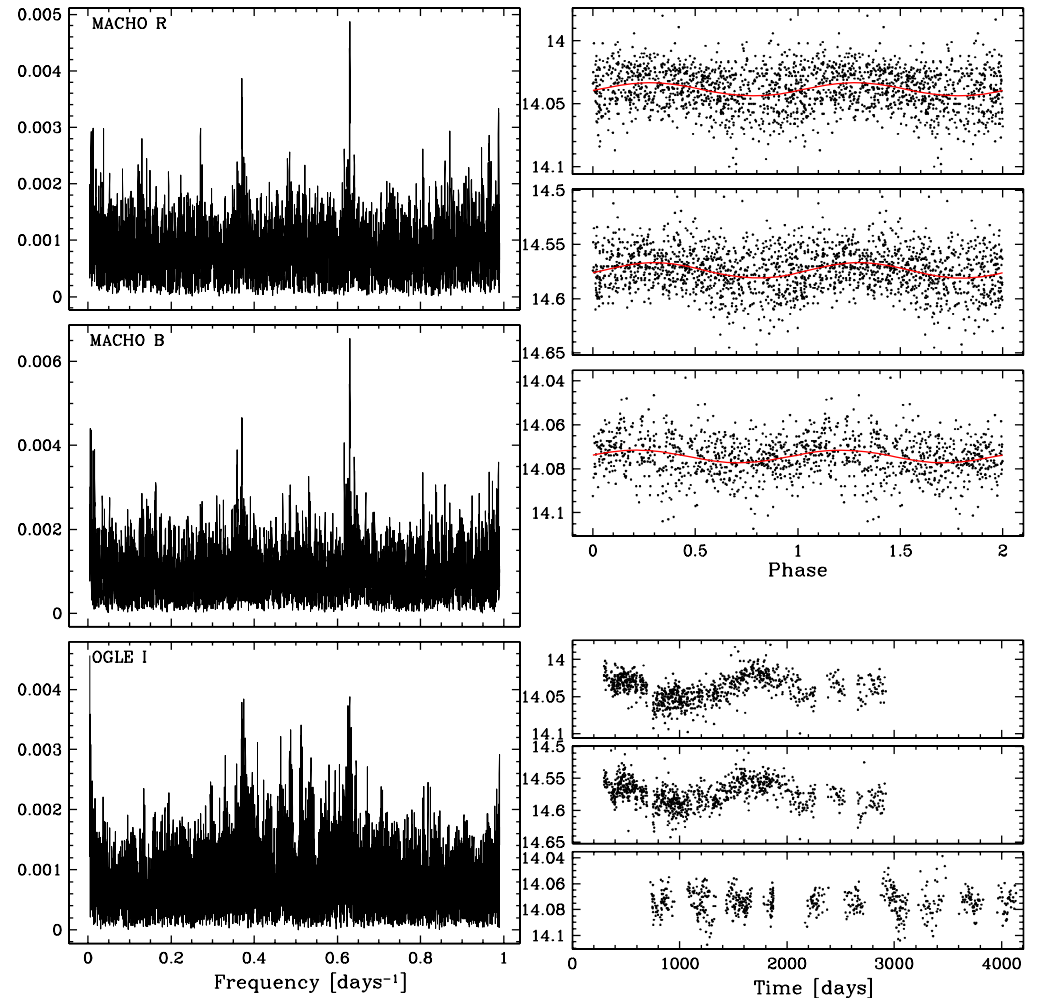

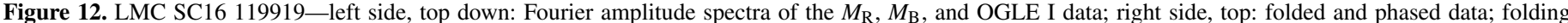
period appears on top; bottom: corresponding data points.

(A color version of this figure is available in the online journal.)
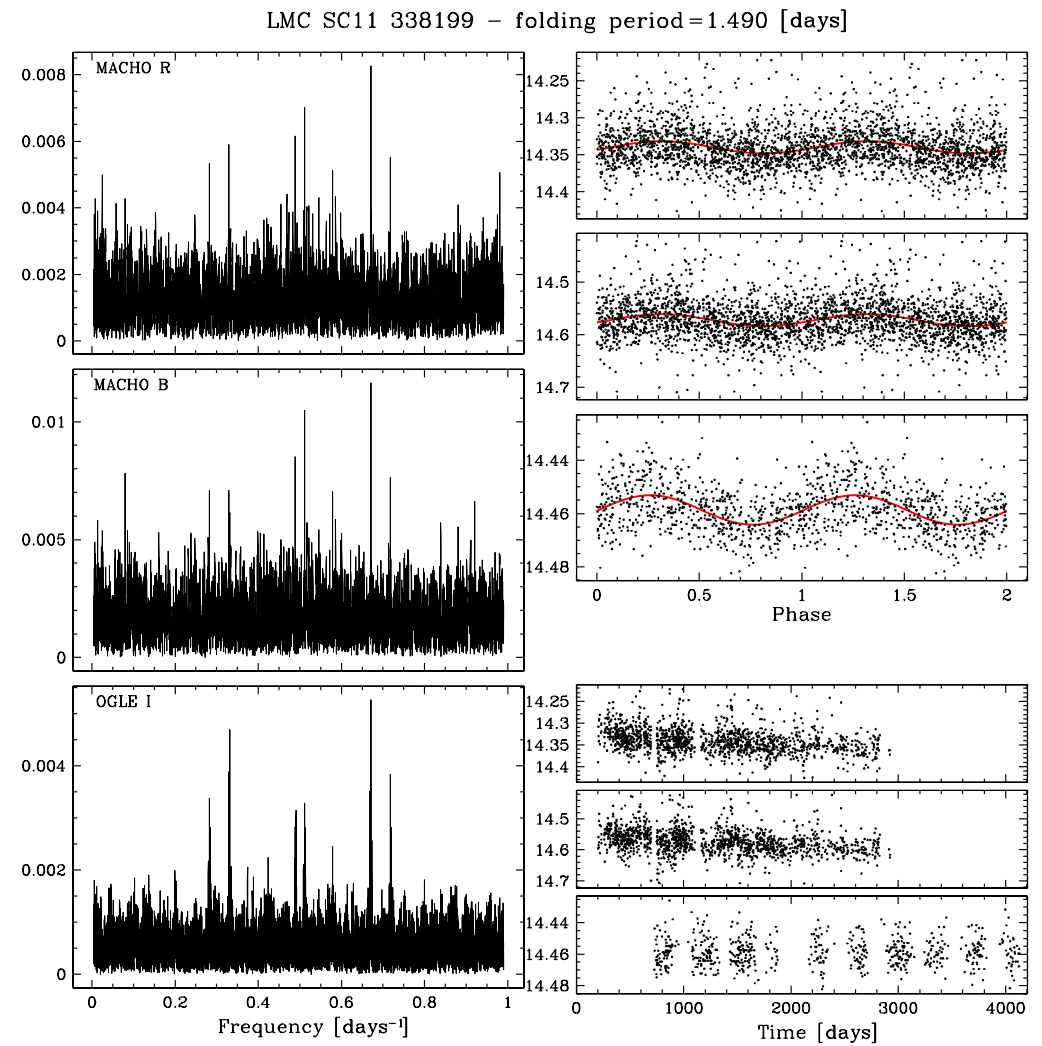

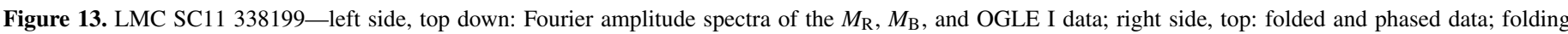
period appears on top; bottom: corresponding data points.

(A color version of this figure is available in the online journal.) 
wrong MACHO object. However, the spectral peaks and the phased, folded light curves suggest otherwise.

The 1.59 day period is much shorter than expected for a Cepheid of similar magnitude and color. Similarly, the long 1380 days is hard to explain. It could be a binary with a very bright companion, although the amplitude of the long period variation would be very large for ellipsoidal motion. The period ratio is also at odds with that of the blue multiperiodic variables that Mennickent et al. (2003) discovered.

4. LMC SC11 338199. This star is multiperiodic with four frequencies that show up simultaneously in $M_{\mathrm{R}}, M_{\mathrm{B}}$, and OGLE I (Figure 13). No other peaks seem to be significant. All four periods are consistent with low-order radial pulsation modes, but it is not obvious why they should all be excited for a star of this brightness. However, there is always the possibility that the light curves of this object are contaminated by a neighboring star, in which case it would fall into a brightness regime where one might expect AI Velorum-type multimode pulsation.

There is recent evidence for proper motion in this star (A. Udalski 2008, unpublished OGLE-III results). If SC11 338199 is a foreground star that would make it even brighter and its nature harder to explain.

\section{SUMMARY}

The Fourier analysis of the light curves of LMC stars in the very broad vicinity of the Cepheid instability strip part yielded 25 new singly periodic and four new multiperiodic variables with ULA ( $\gtrsim 0.01 \mathrm{mag}$ ). The advantage of our approach has been that, for many of these objects, it would be difficult to claim variability on the basis of either the MACHO or the OGLE data only. In addition, we have found RV Tauri and Pop. II Cepheids that do not appear in either the MACHO or OGLE catalogs.

If we include the objects of Buchler et al. (2005), we have 20 ULA objects that fall close to the fundamental (F) and overtone (O) Cepheid PM relations. From Figure 8, it appears that these stars might form separate sequences that are slightly above and parallel to the classical F and O LMC Cepheids. If this effect turns out to be real, it will pose an interesting challenge to explain the nature of these objects. There is evidence that nine of these ULA stars show proper motions and that they could be foreground stars, but that raises other questions as to their nature. Even if we accept them as foreground, this still leaves enough (14 instead of 20) objects to suggest separate PM relations.

Combining this and previous work (Buchler et al. 2005), we now have three stars that are strange Cepheid candidates (Buchler \& Kollath 2001), although the light curve of one of them, SC4 295930, could be contaminated by a very bright red star which would place it erroneously above the PM relation.

Six closely clustered objects fall below the classical Cepheid PM relation (see Figure 8). They could be ULA Pop. II Cepheids or ellipsoidals, although three of them could be foreground stars. A further, very blue object, SC1 330647, could be a ULA W Vir star. Interestingly, a set of binaries appear to lie on essentially a PM relation parallel to that of the RV Tauri/Pop. II Cepheids. Finally, we have uncovered four objects that show multiple modes of ULA.

It is a great pleasure to thank Zoltán Kolláth for providing us with his MUFRAN software. This work has been supported by NSF (AST07-07972 and OISE04-17772) at UF. J.R.B. gratefully acknowledges the hospitality of Mount Stromly Observatory where this work was started. We thank an anonymous referee for his comments which led to a much improved paper.

\section{REFERENCES}

Alcock, C., \& the MACHO Consortium, 1999, PASP, 111, 1539

Beaulieu, J. P., et al. 1995, A\&A, 303, 137

Buchler, J. R., \& Kolláth, Z. 2001, ApJ, 255, 961

Buchler, J. R., \& Kollath, Z. 2002, ApJ, 573, 324

Buchler, J. R., \& Kovács, G. 1987, ApJ, 320, L57

Buchler, J. R., Wood, P. R., Keller, S., \& Soszynski, I. 2005, ApJ, 631, L151

Buchler, J. R., Yecko, P. E., \& Kolláth, Z. 1997, A\&A, 326, 669

Kanbur, S. M., et al. 2003, A\&A, 411, 361

Kolláth, Z. 1990, The Program Package MUFRAN, Konkoly Observatory Occasional Technical Notes No. 1 (Budapest: Konkoly Obs.), http://www.konkoly.hu/staff/kollath/mufran.html

Kovács, G., \& Buchler, J. R. 1987, ApJ, 334, 971

Mennickent, R. E., Pietrzynski, G., Diaz, M., \& Gieren, W. 2003, A\&A, 399, L47

Soszyński, et al. 2002, Acta Astron., 52, 143

Udalski, A., et al. 1999, Acta Astron., 49, 223

Udalski, A., et al. 2008, Acta Astron., 58, 69

Welch, D. L., et al. 1995, in ASP Conf. Ser. 83, Astrophysical Applications of Stellar Pulsation, ed. R. S. Stobie \& P. A. Whitelock (San Francisco, CA: ASP), 232 